\title{
Detection of Brain Tumor Using K-Means Clustering
}

\author{
Ashwini A. Mandwe', Anisa Anjum² \\ ${ }^{1,2}$ Department of Computer Science, Kavikulguru Institute of Technology and Science, Ramtek Dist. Nagpur, India
}

\begin{abstract}
A tumor is a mass of tissue that's formed by an accumulation of abnormal cells. Normally, the cells in your body age, die, and are replaced by new cells. With cancer and other tumors, something disrupts this cycle. Tumor cells grow, even though the body does not need them, and unlike normal old cells, they don't die. As this process goes on, the tumor continues to grow as more and more cells are added to the mass. Image processing is an active research area in which medical image processing is a highly challenging field. Brain tumor analysis is done by doctors but its grading gives different conclusions which may vary from one doctor to another. In this project, it provides a foundation of segmentation and edge detection, as the first step towards brain tumor grading. Current segmentation approaches are reviewed with an emphasis placed on revealing the advantages and disadvantages of these methods for medical imaging applications. There are dissimilar types of algorithm were developed for brain tumor detection. Comparing to the other algorithms the performance of fuzzy c-means plays a major role. The patient's stage is determined by this process, whether it can be cured with medicine or not. Also we study difficulty to detect Mild traumatic brain injury (mTBI) the current tools are qualitative, which can lead to poor diagnosis and treatment and to overcome these difficulties, an algorithm is proposed that takes advantage of subject information and texture information from MR images. A contextual model is developed to simulate the progression of the disease using multiple inputs, such as the time post injury and the location of injury. Textural features are used along with feature selection for a single MR modality.
\end{abstract}

Keywords: Image Segmentation, K-Means clustering, MRI, Tumor Detection. Abnormalities, Brain tumor, Fuzzy C-means, k-means, Magnetic Resonance Imaging (MRI)

\section{Introduction}

No one knows what causes brain tumors; there are only a few known risk factors that have been established by research. Children who receive radiation to the head have a higher risk of developing a brain tumor as adults, as do people who have certain rare genetic conditions such as neurofibromatosis or Li-Fraumeni syndrome. But those cases represent a fraction of the approximately 35,000 new primary brain tumors diagnosed each year. Age is also a risk factor -- people over the age of 65 are diagnosed with brain cancer at a rate four times higher than younger people.

A primary brain tumor is one that originates in the brain, and not all primary brain tumors are cancerous; benign tumors are not aggressive and normally do not spread to surrounding tissues, although they can be serious and even life threatening. The National Cancer Institute estimates there will be about 23,380 new cases of brain cancer diagnosed in 2014.

A tumor is a mass of tissue that's formed by an accumulation of abnormal cells. Normally, the cells in your body age, die, and are replaced by new cells. With cancer and other tumors, something disrupts this cycle. Tumor cells grow, even though the body does not need them, and unlike normal old cells, they don't die. As this process goes on, the tumor continues to grow as more and more cells are added to the mass.

Primary brain tumors emerge from the various cells that make up the brain and central nervous system and are named for the kind of cell in which they first form. The most common types of adult brain tumors are gliomas and astrocytic tumors. These tumors form from astrocytes and other types of glial cells, which are cells that help keep nerves healthy.

The second most common type of adult brain tumors are meningeal tumors. These form in the meninges, the thin layer of tissue that covers the brain and spinal cord.

Radiologists examine the patient physically by using Computed Tomography (CT scan) and Magnetic Resonance Imaging (MRI). MRI images showed the brain structures, tumor's size and location. From the MRI images the information such as tumors location provided radiologists, an easy way to diagnose the tumor and plan the surgical approach for its removal.

This image processing consist of image enhancement using histogram equalization, edge detection and segmentation process to take patterns of brain tumors, so the process of making computer aided diagnosis for brain tumor grading will be easier.

\section{Objective}

- Enhanced information about brain tumor detection and segmentation.

- Deep Study of Techniques like performing a biopsy, performing imaging, like taking a MRI or CT scan of the brain will be done.

\section{Problem Statement}

The brain is the anterior most part of the central nervous system. Brain tumor is an intracranial solid neoplasm. 


\section{International Journal of Science and Research (IJSR) \\ ISSN (Online): 2319-7064}

Index Copernicus Value (2013): 6.14 | Impact Factor (2015): 6.391

Tumors are created by an abnormal and uncontrolled cell division in the brain. In this work, we have used axial view of the brain image (2D) from MRI scan because MRI scan is less harmful than CT brain scan. A patient is subjected to different diagnostic methods to determine the cause of the symptoms mentioned by him. Techniques like performing a biopsy, performing imaging, like taking a MRI or CT scan of the brain will be done.

Traditional method in hospitals is to segment the medical image under consideration, manually and this depends on how well the physician can perceive the image under consideration to get the required region extracted out, which is made difficult because of minute variations and resemblance between the original and affected biological part in the image. The shortage of radiologists and the large volume of MRI to be analyzed make these readings labor intensive and also cost expensive.

It also depends on the expertise of the technician examining the images [10]. Estimates also indicate that between 10 and $30 \%$ of tumors are missed by the radiologists during the routine screening. The existing method is based on the threshold and region growing. At the threshold based segmentation the image is considered as having only two values either black or white.

But the bitmap image contains 0 to 255 gray scale values. So it ignores the tumor cells also. In [1] case of the region growing based segmentation it needs more user interaction for the selection of the seed. Seed is nothing but the center of the tumor cells; it may cause intensity in homogeneity problems.

\section{Literature Survey}

This section deals with the works related to the brain tumor detection and segmentation in medical images. Somkantha, et al [1] designed a new edge following technique for boundary detection in noisy images and applied it to object segmentation problem in medical images.

The proposed technique was applied to detect the object boundaries in several types of noisy images where the illdefined edges were encountered. Gooya, et al [2] presented a method GLISTR for segmentation of gliomas in multimodal MR images by joint registering the images to a probabilistic atlas of healthy individuals.

The major contribution of the paper was the incorporation of tumor growth model to adopt the normal atlas into the anatomy of the patient brain. Parisot, et al [3] contemplated a different approach for detection, segmentation and characterization of brain tumors. This technique exploits prior knowledge in the form of a sparse graph delineating the expected spatial positions of tumor classes. In this paper, implied a novel way to encode prior knowledge in tumor segmentation, making use of the fact that the tumors tend to appear in the brain in preferential locations. They combined an image based detection scheme with identification of the tumor's corresponding preferential location, which was associated with a specific spatial behavior. Manikis, et al [4] suggested a novel framework for assessing tumor changes based on histogram analysis of temporal Magnetic Resonance Image (MRI) data.

The proposed method detects the distribution of tumor and quantitative models its growth or shrinkage offering the potential to assist clinicians in objectively assessing subtle changes during therapy. Bauer, et al [5] determined a novel approach to adapt a healthy brain atlas to MR images of tumor patients. They presented a new method which makes use of sophisticated models of bio-physio mechanical tumor growth to adapt a general brain atlas to an individual tumor patient image.

An effective modified region growing technique for detection of brain tumor. Modified region growing includes an orientation constraint in addition to the normal intensity constrain (Weaver et al., 2012). The performance of the technique is systematically evaluated using the MRI brain images received from the public sources. A Self-Organizing Map (SOM) is a competitive artificial neural network with unsupervised learning.

To increase the SOM learning effect, a Fuzzy-Soft Learning Vector Quantization (FSLVQ) algorithm has been proposed in the literature, using fuzzy functions to approximate lateral neural interaction of the SOM Wen-Liang Hung., 2011). In 1985, Computational performance of FSLVQ is still not good enough, especially for large data sets. In this work, we propose a suppressed FSLVQ(S-FSLVQ) using suppression with a parameter learning schema. We then apply the SFSLVQ to MRI segmentation and compare it with several existing methods.

\section{Proposed System}

We have proposed segmentation of the brain MRI images for detection of tumors using clustering techniques. A cluster can be defined as a group of pixels where all the pixels in certain group defined by a similar relationship [1]. Clustering is also known as unsupervised classification technique. The name unsupervised classification because the algorithm automatically classifies objects based on user given criteria. Here K-means clustering algorithm for segmentation of the image followed by morphological filtering is used for tumor detection from the brain MRI images. The proposed block diagram is as shown.

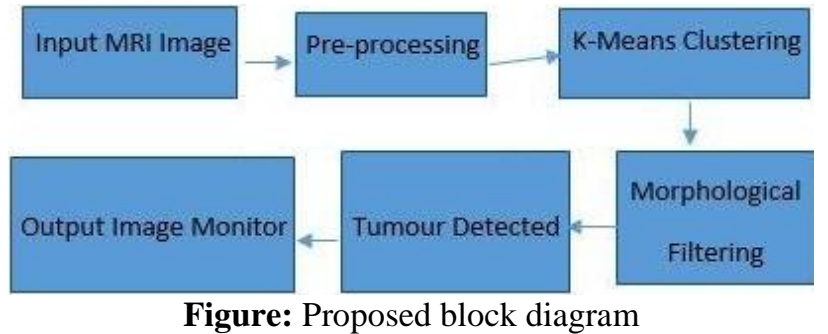

The preprocessed image is given for image segmentation using K-means clustering algorithm. As there are chances of occurrence of misclustered regions after the application of K-means clustering algorithm.

\section{Volume 5 Issue 6, June 2016 www.ijsr.net}




\section{International Journal of Science and Research (IJSR) \\ ISSN (Online): 2319-7064}

Index Copernicus Value (2013): 6.14 | Impact Factor (2015): 6.391

\section{Module Details}

The data is first presented according to example pictures of the image processes involved in the system. As discussed in the previous design part, the image used is of grayscale MRI brain scans.

First we acquire the images and pre-processes it under a median filter. These are some of the examples the images:

Next the edges of the images are specified with the sobel edge detection. The images processed are as follows:

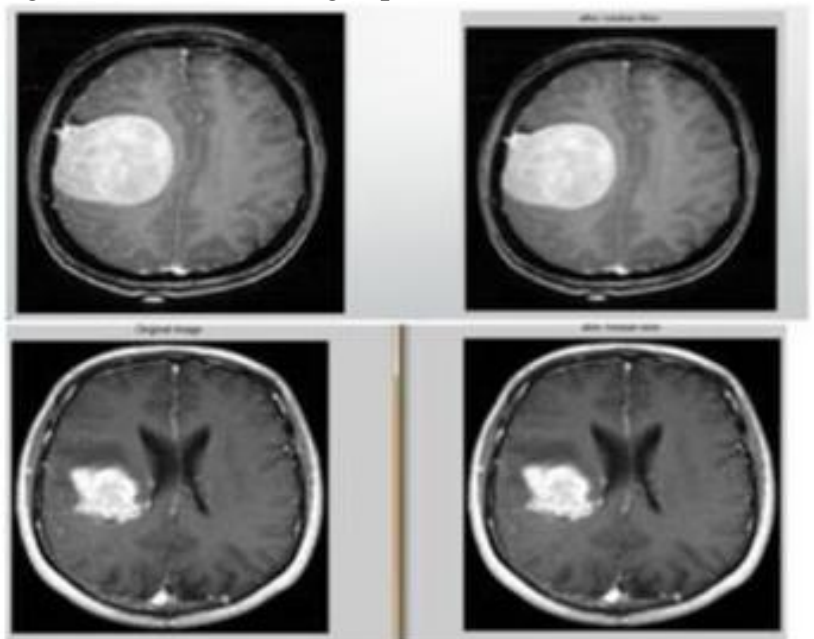

Fig 1: example of MRi scan image before (left) and after (right) applying the median filter

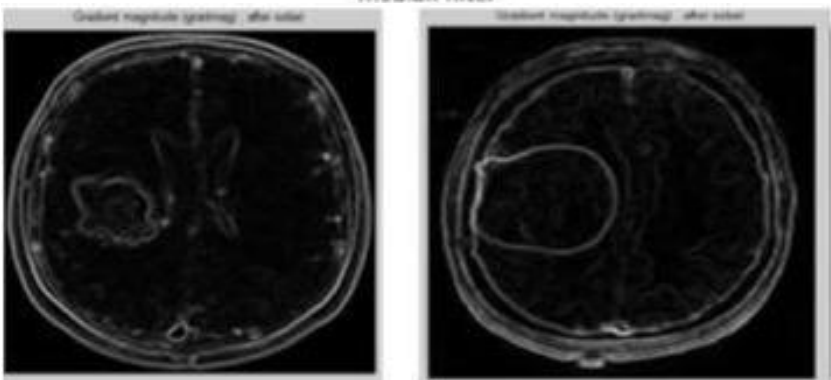

Figure 2: Images after sobel edge detection algorithm is applied

Later the images would go through a Modified Histogram Clustering - Color Threshold technique to identify the position of the tumor. According to various MRI scan images, the threshold values of the image's HSV for the tumor happens to be common and lies between these values:

Table 1: Threshold values applied

\begin{tabular}{|l|l|l|}
\hline HSV & High $(\%)$ & Low $(\%)$ \\
\hline Hue & 35 & 0 \\
\hline Saturation & 75 & 0 \\
\hline Value & 100 & 70 \\
\hline
\end{tabular}

The values are set as a threshold and applied with convolution with the original image to determine the location of the tumor. Through the syntax of bwlabel() on Matlab, each block of image found is subjected to a value of label. This allows us to gain property information about the block using the improps() syntax. The area can be found and displayed.

\section{Conclusion}

Segmentation of brain image is imperative in surgical planning and treatment planning in the field of medicine. In this work, we have proposed a computer aided system for brain MR image segmentation for detection of tumor location using $\mathrm{K}$ - means clustering algorithm. The noise free image is given as input to the $\mathrm{k}$-means and tumors are extracted from the MRI image. The performance of brain tumor segmentation is evaluated based on K-means clustering. Dataset consists of Magnetic Resonance Imaging (MRI) size of $181 X 272$. The MRI image dataset that we have utilized in image segmentation technique is taken from the publicly available sources. The brain image dataset is divided into two sets. Training dataset and testing dataset. Thus, the pre-processing is done by filtering. Segmentation is done by advanced K-means algorithm and fuzzy c means algorithm. Feature extractions is done by threading and finally, approximate reasoning method to recognize the tumor shape and position in MRI image using edge detection method.

\section{References}

[1] Vida Harati, RasoulKhayati, AbdolrezaFarzan, "Fully automated tumor segmentation based on animproved fuzzy connectedness Algorithm in BrainMR Images", Elsevier Ltd,Vol 7, pag: 483-92, May 2011.

[2] Ali Gooya, George Biros Christos Davatzikos, "An EM Algorithm for BrainTumorImagesRegistration: A Tumor Growth Modling Based Approach", IEEE,Vol 2, pag: 375-90, May 2010

[3] Akansha Singh , Krishna Kant Singh, "A Study Of Image Segmentation Algorithms For Different Types Of Images", International Journal of Computer ScienceIssues, vol. 7,Issue 5, pp 414-417,2010.

[4] Mansur Rozmin, Prof.ChhayaSuratwala, Prof.VandanaShah,'Implementation Of Hard C-Means Clustering Algorithm For Medical Image Segmentation", Journal Of Information Knowledge and Research in Electronics and Communication Engineering,vol.2, no.2, pp 436-440,Nov12-Oct13.

[5] T.N. Pappas. An adaptive clustering algorithm for image segmentation. IEEE T. Signal Process., 40:901 $914,1992$.

[6] Fan, J., Han, M. and Wang, J. Single point iterative weighted fuzzy c-means clustering algorithm for remote sensing image segmentation. Pattern Recognition, 42(11), pp. 2527-2540., 2009.

[7] David Rivest-Henault, Mohamed Cheriet, "Unsupervised MRI segmentation of brain tissues using a local linear model and set,"Elsevier, 2011.

[8] T.Logeswari, M.Karnan, "Hybrid Self Organizing Map for improved Implementation of Brain MRI Segmentation,'IEEE, 2010.

[9] T. Logeswari, M.Karnan, “An Improved Implementation of Brain [A.Alexandra Constantin, B.Ruzena Bajcsy, C.Sarah Nelson, "UnsupervisedSegmentation of Brain Tissue inMulitvariate MRI,’IEEE, 2010.

[10]El-Sayed Ahmed El-Dahshan, Tamer Hosny, AbdelBadeeh M.Salem, "Hybrid intelligent techniques for MRI Brain Images classification,” Elsevier ltd, 2009.

\section{Volume 5 Issue 6, June 2016 www.ijsr.net}




\section{International Journal of Science and Research (IJSR) \\ ISSN (Online): 2319-7064}

Index Copernicus Value (2013): 6.14 | Impact Factor (2015): 6.391

[11] Tumor Detection using Segmentation Based on Hierarchical Self Organizing Map," IEEE, 2010.

[12] Ehab F.Badran, Esraa Galal Mahmoud,and Nadder Hamdy, "An Algorithm for Detecting Tumors in MRI Images,"IEEE, 2010.

[13] Ali Gooya, George Biros Christos Davatzikos, “An EM Algorithm for Brain Tumor Images Registration:A Tumor Growth Modling Based Approach,” IEEE,2010.

[14] S.Taheri, S.H.Ong, V.F.H. Chong, "Level-set segmentation of brain tumors using a threshold-based speed function, "Elsevier, 2009.

[15] Brijesh Shah, Satish Shah, Y P Kosta,'Novel Improved Fuzzy C-Mean Algorithm MR-images Segmentation,'IJSCE,2010and L. Avila, Eds., (ser.Lecture Notes in Computer Science), vol. 6453 Heidelberg, Germany: Springer, 2010, pp. 190-198.

Volume 5 Issue 6, June 2016 www.ijsr.net 